\title{
The cultural logic of Shilin Yi nationality Folk Therapy
}

\author{
Zhao Ying \\ Southwest Minzu University, Southwest Institute for Nationalities
}

Keywords : Shilin Yi Nationality, folk therapy practice, cultural logic.

\begin{abstract}
Shilin Folk Therapy of YI Nationality, as a concentrated expression of the ethnic culture of Chinese medicine, has a distinct regional and national character . In the field of medical anthropology, folk therapy has both religious and cultural values. and that emphasizing the importance of "harmonious relationship between patients and healers" and "believable explanation for what causes illness". Through extensive fieldwork, the author finds that Shilin Yi nationality folk therapy practice is closely related to the relationship between relatives and social network. The physical, mental, and social efficacy are significant factors in driving local people to believe and adopt folk therapy. In this paper, the author thinks that Folk therapy has a profound influence on the Yi nationality and the local society.
\end{abstract}

\section{Introduction}

Folk therapy has some influence on human society, economy, culture and other aspects. Because of the advancement of modern medicine and the development of biotechnology, the system of modern medicine has been gradually established and further improved. Meanwhile, folk therapy organically integrates with the modern medical system, and presents diversified medical development trend. In contemporary society, due to the consideration of cultural values and religious beliefs, people have different choices of medical therapies.

According to the cases in traditional Chinese medicine and pharmacy, sociology and other subjects, Chinese scholars have profoundly explored the minority nationality medicine and folk medicine. Those research results are widely being adopted to the actual medical practices, and bring about constructive and instructional suggestions for medical practices. The studies of Chinese scholars on the minority nationality medical practices pay great attention to the investigation of the minority nationality medicine. They specifically collect various therapies of the minority nationality medicine, and highlight the method with the combination between theories and practices. They give some explanation of the present situation and coming crisis of the minority nationality medicine, analyze the existing problems in practical minority nationality medicine, and attempt to put forward relevant countermeasures and suggestions. On the basis of "Things between Earth and Sky", "Le e Te Yi”, “ The History of Yi Nationality in Southwest of China”, "Ming Dynasty Yi Medicine Manual”, "Medical History of Yi Nationality”, "Yuanyang Yi Medicine Manual”, "Shuangbai Yi Medicine Manual”, "Yibing Shu”, "Wa Wa Sheng Cheng Shu”, "Classic of Offering Sacrifices and Dedicating Medicine" and other literature of Yi nationality, Meng Zhiren[1] and Ma Weiguang[2] verificined the Yi medicine history of over 200 years. They made specific division of the historical stages, and briefly introduced the relative contents of the medical classics of Yi nationality. Also they affirmed to the contribution of Yi medicine to surgery, orthopedics, internal medicine, gynecology, pediatrics and other aspects. Zhu Guoxiang and $\mathrm{Xu}$ Junfei[3] made a relevant introduction to the versions and contents of a series of Guizhou medical literature "Qi Gu Shu". And they made the explanation from five aspects, including the "Wuti" theory in Yi medicine, the simplicity of Yi medicine, the theoretical relationship between Yi medicine and traditional Chinese medicine, the curative effects of prescriptions, and the maturity of prescriptions. Cheng Yaqun and Ma Kebu[4], from the perspective of the history of myth and legend of Yi medicine, focused on the analysis of the origin of Yi medicine, and mainly argued the medicine origin from instinct, the medicine origin from labour, the medicine origin from thought and witchcraft, and the medicine 
origin from sages. Zhang Zhidao[5] considered that the origin of Yi medical theories is composed of the holistic view of the concept that man is an integral part of nature, zangfu and meridians theory centered by the five elements, the $\mathrm{Ai} \mathrm{Pu}$ theory, the principle of eight diagrams, and some other theories. Xu Jiapeng and Yang Benlei[6], and Liu Yuan[7] et al.respectively made investigation on Yi medicine in Chuxiong Prefecture of Yunnan Province and Liangzhou Prefecture of Sichuan Province, to elaborate the present situation of the development of Yi medicine. And they provided relevant countermeasures and suggestions according to the existing problems, to promote the further development of Yi medicine. From the dimension of cultural values, Wang Dan[8] analyzed that folk therapies of Baima Tibetan have physiological and psychological features, so as to analyze the relationship between easing suffering and the social network relation between the relatives and themselves. However, it neglects the elements of religious beliefs in folk therapies. Thus, this paper takes the example of Shilin County in Yunnan Province to make a case study, to make explanation according to the relationship between the folk therapies of Yi medicine and religious beliefs, trying to explain the influence of folk therapies on the society of Yi nationality.

\section{Bimo in Yi Nationality and its medical practice}

Shilin Yi Autonomous Country, with the old name of "Lunan", is a suburban county in Kunming City Yunnan Province. It is 78 kilometers from Kunming. County-wide total area is 1719 square kilometers. Jurisdiction is over 7 towns and 1 township. In the whole county, there are more than twenty minorities which are mainly Yi nationality, Miao nationality, Zhuang nationality. The population of Yi nationality accounts for $97.4 \%$ of the nationality population. Within Shilin County, Yi nationality has "Sani”, "Black Yi”, "Yiqin”, “Axi”, “Ayizi” and other branches. Bimo originated from the priests or chiefs in the period of Yi nationality's patriarchal clan society. They are the presiders of the primitive religious sacrificial rites of Yi nationality, and the important disseminator and inheritors of Yi nationality's traditional knowledge culture. Yi nationality believes in ghosts and gods, and Bimo plays the role to communicate between people and ghosts and gods. The breakdown of Tusi regime makes Bimo lose its dependent power, but turns into the folk to engage in worship ceremonies and other ritual activities. Bimo culture is the synthesis of various kinds of witchcraft and classics in Yi language. Its origin is mainly oriented by the beliefs of souls or ghosts and gods. When people meet things and doubt how to make decision, no matter the things are big or small, they would employ a sorcerer to take auspices by chicken bones. That is the main activity of Bimo.[9]

\section{Bimo in Yi Nationality}

Bimo is the transliteration of Yi language. "Bi" refers to chanting, and "mo" is the honorific title of the knowledgeable elders. In the process of Yi nationality's historical development, Bimo has very important function. In ancient seignorial system period of Yi nationality, Bimo was not only the person who took the charge of instruments and lead religious ceremonies, but also acted as teachers, advisers, doctors and judges. Meanwhile, they were also intellectuals who created characters, composed and collected Yi classics, and thoroughly understand the history, astronomy, and geography of Yi nationality. Bimo of Yi nationality was limited in males, and had strict hereditary system.

In the life of Yi people, Bimo enjoyed a high position and played significant role. Bimo was the inheritor of Yi culture. In the long history of Yi nationality, Bimo once undertook the role of doctors. Bimo combined witchcraft and leechcraft, and utilized both spirit and medicine, to make people feel their leechcraft "scared and efficacious". In addition, Bimo also acted as a musician. The first factor of Bimo is ability to sing the scriptures which would be sung in a variety of worship activities. Bimo has his own special clothing and sacrificial instruments. During the worship ceremonies, Bimo would be chanting the scriptures while shaking the sacrificial bells, so as to transfer the information between human and ghosts and gods to boost the sacrificial stateliness. The sacrificial instruments used by Bimo in worship ceremonies contains sacrificial hats, sacrificial clothes, sacrificial bells, sacrificial fans, sacrificial boxes or sacrificial bags, and sacrificial cylinders. 


\section{Medicine Literature of Yi Nationality}

According to incomplete statistics, there are more than 20 existing ancient books of Yi medicine, mainly including "Zuoji Xianyao Gongsheng Jing”, "Shuangbai Yi Medicine Manual”, "Yuanyang Yi Medicine Manual", "Yibing Shu”, "Haoyao Yibing Shu”, "Yi Classic of Dedicating Medicine", "Wa Wa Sheng Cheng Shu", "Salvation Book: Book of Taking Good Medicine", "Classic of Offering Sacrifices and Dedicating Medicine", "Nie Su Nuo Qi", "Yi Medicine of Ailao Mountains", and so on. Publishments in recent years also include "Yi Medicine Records", "Animal Medicine of Yi Nationality", "Botanical Medicine of Yi Nationality", "Precious Materia Medica of Yi Medicine”, "Records of Yi Medicine books", "Directory of Yunan Ethnic Drugs", "Yi Medicine of Eshan", "Medical History of Yi Nationality”, and some relevant works of Yi medicine. From those works of Yi medicine, it can be seen that Yi medicine has its unique methodicalness and system. It combines the cosmology that man is an integral part of nature, to found distinctive national medicine theories. Bimo also made the single prescription and compound prescription of Yi medicine spread to later generations by means of writing characters. For example, Yi medicine "Qizha” (metapanax delavayi, as unique medicine of Yi nationality) is used to treat various eye diseases. "Aji” (pyracantha crenulata, as unique medicine of Yi nationality) is adopted to treat altitude reaction, gastrointestinal ulcer and traumatic bleeding. "Aniushitu” (actinidia lindl, as unique medicine of Yi nationality) is applied to treat liver cirrhosis. "Shibu" (cassiope selaginoides, as unique medicine of Yi nationality) is utilized to treat rheumatoid arthritis. All of them have special curative effects.[10]The categories of Yi medicine are countless. According to the recordation in "The Medicine and Pharmacology of Yi Nationality", the included medicines reaches 1189 in which there are 871 botanical medicines, 262 animal medicines, and 56 mineral medicines.[11]

\section{The Participation of Kinship and Social Relationship in Practice of Yi Medicine}

The experience of indisposition and treatment of Yi nationality in Shilin can be seen as an ordinary life experience. In the local stockaded villages of Yi nationality, everyone establishes their kinship relation with each other through blood relationship, affinity, or "claiming nominal kinship", so as to form an inseparable relationship within the groups. From marriages, funerals, house-building and beam-suspending, treatment, sacrificial activities, and other daily life, it can be seen the influence of kinship relation. "Claiming nominal kinship" is a new way to extend the range of identity and try to establish a new kinship relation network, based on those who have no blood or affinity relationship. The establishment of social network relationship of Sani people in Yi nationality in Shilin not only includes the kinship relation based on blood, affinity and geographical relationship, but also contains the group identification and mutual mechanism which is built by the playmates, childhood friends, and other friend and partner relationship based on the age level. The pain brought by the diseases should originally be born by individuals or families independently. However, because of the establishment of local kinship relation and social relationship network, the treatment of indisposition becomes the event of group participation. In the stockaded villages of Yi nationality in Shilin, the treatment ceremonies of Bimo have multi-effects of treatment, evocation, exorcism, and so on.

For example, if someone is confused and stupefied, fears and palpitates, feels hypodynamia, has no appetite, and has some other symptoms, others would think he lost his soul. And the evocation ceremony should be held to make him cured. In the concept of Sani people of Yi nationality, "the soul attaches to the body and acts with the body. But if the soul gets a fright, it would leave away from the body."[12] The reasons of losing soul are various, which can be specifically divided into meeting new graves, meeting venomous serpents and wild beasts, passing in front of mountain temples, falling into water, and so on. Thus, according to the different reasons of losing soul, Bimo would go to different places to call back the soul. The common places are new graves, big mountains and huge stones, pools, doorways, and so on.

"Lepu Birao is calling back the soul to our homeland. Mountains and stones are in front. The big mountain lives mountain dragon. The thick forest lives forest dragon. Don’t be frightened if the 
soul see them. Come back, the soul, come back. The mountain is the one greeting the soul. The forest is the one welcoming the soul.

The soul returns in front of the tree. The treetops reach to the sky. The tree branches diverge in the air. The leaves look like cloud clusters. The tree trunk is straight and round. The tree array is neat and uniform. Don't fear, the soul, don't fear. Come back, the soul, come back. This is the tree in home. Trees in alien lands can't cover the soul in our home. Only trees in local lands can cover the soul in our home.

Lepu Birao is calling back the spirit of the dead to the lake. The lake is wide to surge waves. And the waves are persistent, regardless of the daytime and night. Don't fear, the soul, don't fear. It is the lake calling for soul. It is the lake welcoming people.

The soul returns to the farmland. The farmland is wide and plane. The sound of evocation spreads far and wide, throughout every corner of the farmland. The farmland is the one cultivated by you. The farmland is the one seeded by you. Come back, the soul, come back. The soul is called back to the edge of the village. The twelve stockaded villages are bubbling with noise from both men and women. They shout and vociferate. Don't fear, the soul, don't fear. They are men welcoming the soul. They are women evocating the soul.

The soul is called back to the middle of the village. In the twelve stockaded villages, the dog constantly barks, the barking rising here and subsiding there. Don't fear, the soul, don't fear. That is the dog welcoming the soul. That is the dog evocating the soul.

The soul is called back to the home. In the courtyard in front of the door, the dog nods its head and wags its tail. The dog is very intimate with the soul. It welcomes the soul to return.

The soul is called back to the threshold. The threshold has two pieces of wood. The facade has four pieces of board. The facade is decorated by gold. The threshold is decorated by silver. The lintel is carved with flying dragons. The magnificent door is opening. The soul is pleased to enter. People are also pleased to enter.

The soul is called back to the central room. The golden pheasant is spreading the wings and craning the neck to crow. The crow is loud and clear. The gold pheasant is welcoming the soul to return.

The soul is guided in front of the pillar. The head of the pillar is the Shuoshu Lu. It is the evocating Lu. It is the protecting Lu. It is the herding Lu. It is the crop Lu. The soul depends on the Lu to joy. People relies on the Lu to joy.

The soul is guided in front of the kitchen. The basins, the bowls and the spoons are all in readiness. Mother and daughters take the charge of cooking. Aerial fog seeped around the cooking bench. The breakfast is already cooked. The supper is already cooked. The delicious food is enjoyed by the soul. And people are also sated with the food. That gourd of water is enjoyable for the soul, and quench people's thirst. Zao Za Guo Si Pa. Zhi Nan Shu Si Ma. Eat here when hungry. Drink here when thirst.

Come back. Come back. Does the soul come back? It does come back.[13]

In different places, the ceremonies of evocation would be held differently. The ultimate goal is to call back the soul of the person who loses the soul, so as to bring physical health.

The elders in Yi stockaded villages in Shilin mostly suffer from diseases of different degree. This is connected with the elder's past life background, individual experiences and present worries. From the view of local people, only when the elders are in critical situation would they choose to go to hospital to treat. This is not simply because the medical bills are too expensive to afford, or the unavailability of the treatment in hospital, but because treatment in the stockaded villages can be accompanied and helped by relatives and friends.

\section{The cultural logic of Yi therapy practice in Shilin}

Yi therapy practice in Shilin is embedded in the kinship and social relationship network. For the same symptoms, the process of treatment may be different. For instance, in the case that the hospital treatment is effective for Grandma $\mathrm{Bi}$, she still chose to return to the stockaded village to treat the paralysis by herbal medicine. Meanwhile, she asked for Bimo to hold evocation and other worship 
ceremonies to realize the functions of warding off diseases and removing ill fortune. The involves the mystery of the cultural logic of the therapy practice of Sani people in Yi nationality in Shilin. Though this kind of cultural logical choice is closely related to the doctor-patient relationship, hospitalization costs and even the cause explanation of the diseases, it cannot be separated from the kinship relation and social relationship. The therapy practice of Yi nationality in Shilin is to rationally partake the pain brought by diseases through mobilizing and depending on the relative and social relationship, so as to promote the harmony and stability of the social relationship.

In modern therapy system, the cause explanation of diseases is usually proposed by doctors or medical technologists. In stockaded villages in Yi nationality in Shilin, because villagers stay in the acquaintance society and common social network relationship for a long time, same disease symptoms can be given different cause explanation due to the individual differences to carry out different treatment process. Within the internal social groups in the stockaded villages, people seek for folk therapy methods. This attributes to their belief in Bimo and folk therapy methods. Specific cause explanation of diseases depends on the relatives and families or friends in the social relationship to make. The formation of social network relationship plays a vital role in the cause explanation of diseases and even the choice of therapies.

Sani people in Yi nationality in Shilin would independently choose the therapy methods according to their own experiences of the diseases. With the progress of modern medicine, though people recognize the timeliness and effectiveness of hospital medicine, they would still choose traditional therapy practice methods to get treatment. Through traditional therapy practice methods, the pains of patients can find comfort from the consolation of groups. Those "unmentionable diseases" in reality which cannot be open to the public would be transformed to the publicized and shared diseases. This is the reason why Sani people in Yi nationality prefer to choose herbal therapies of traditional Chinese medicine to get treatment in the case that Chinese herbal medicine has faster curative effects than modern western medicine.

The therapy practice of Yi nationality in Shilin has multiple effects of physiology, psychology and sociality. Patients' choice of therapy methods not only simply pursues the physical health, but also seeks for the comfort at the psychological and social levels. The medical process of Sani people in Yi nationality in Shilin is not only restricted to the individual and family choices to seek therapy, but is a sort of therapy practice involving the participation of social groups.

\section{Acknowledgments}

National Social Science Fund West Project (NO.13XMZ067);2014 National Social Science Foundation Project (NO.14ZDB119).

\section{References}

[1]Meng Zhiren. A Brief Review on the History of Yi Medicine. Social Sciences In Yunan. 1992(5).

[2]Ma Weiguang. A Summarization of Yi Medicine. Journal of Yunnan College of Traditional Chinese Medicine. 1990(9).

[3]Zhu Guoxiang, Xu Junfei. The Cultural Characteristics of Yi Medicine from the Medical Literature: Qi Gushu. Journal of Sichuan Minzu College. 2015(6).

[4]Cheng Yaqun, Ma Kebu. The Origin of the Yi Medicine-From the Angle of Culture of Traditional Chinese Medicine (myth). Journal of Xichang College (Social Science Edition). 2008(3).

[5]Zhang Zhidao. Exploration on the Origin of Yi Medicine. Journal of Yunnan College of Traditional Chinese Medicine (Supplement). 2008(6).

[6]Xu Jiapeng, Yang Benlei. Investigation of the development status of Yi Medicine in Chuxiong Prefecture. Journal of Yunnan Traditional Chinese Medicine. 2009(10).

[7]Liu Yuan, Azi Ayue, Liu Chao, Peng Lianxin, Shang Yuanhong, Meng Qingyan. The Reports of Yi Nationality Medicine of Liang Shan of Sichuan Province. Lishizhen Medicine And Materia 
Medica Research. 2006(8).

[8]Wang Dan. Sharing and Participation: Cultural Logic of Folk Medicine Practice of Baima Tibetan. Ethno-National Studies. 2013(6).

[9]Xu Ming. Research on Bimo Culturalism. Journal of Southwest University for Nationalities (Philosophy and Social Science Edition). 1989(3).

[10]Sha Xuezhong. Significance and Value of the Study on Bimo Medicine Experience in Liangshan. Laboratory Medicine and Clinic. 2010(1).

[11]Guan Xiangzu. The Medicine and Pharmacology of Yi Nationality. Yunnan Nationalities Publishing House. 1993.

[12]Ethnic and Religious Affairs Bureau of Shilin Yi Nationality ed. The Translation and Reorganization of Sani Sacrificial Lines of Yi Nationality. Yunnan Nationalities Publishing House. 1999(12):93.

[13]Ethnic and Religious Affairs Bureau of Shilin Yi Nationality ed. The Translation and Reorganization of Sani Sacrificial Lines of Yi Nationality. Yunnan Nationalities Publishing House. 1999(12):89-91. 